http://dx.doi.org/10.1590/1678-4162-9296

Arq. Bras. Med. Vet. Zootec., v.69, n.3, p.543-550, 2017

\title{
Resposta hematológica de cavalos de enduro, que correram diferentes distâncias, no período pós-prova
}

\author{
[Hematologic response of endurance horses that ran different \\ distances in the post race period]
}

\author{
R.F. Siqueira, W.R. Fernandes
}

Faculdade de Medicina Veterinária e Zootecnia - Universidade de São Paulo (FMVZ-USP) -São Paulo, SP

\section{RESUMO}

O objetivo deste trabalho foi estudar as alterações hematológicas após provas de enduro de diferentes distâncias até 14 dias após a prova, período de recuperação metabólica e musculoesquelética, as quais ocorrem em cavalos treinados e que concluíram as competições em boas condições de saúde. Foram avaliados 32 cavalos Árabes, com $400( \pm 32 \mathrm{~kg})$, treinados para provas de enduro, que competiram em quatro provas nas categorias velocidade livre de $80 \mathrm{~km}(\mathrm{n}=13), 120 \mathrm{~km}(\mathrm{n}=14)$ e $160 \mathrm{~km}(\mathrm{n}=5)$. Amostras de sangue foram coletadas antes da largada (M0), imediatamente após a saída da última inspeção veterinária (M1) e três horas após (M2). Já nos respectivos haras, foram coletadas amostras aos três (M3), sete (M4) e 14 (M5) dias após a prova. Houve aumento dos valores de eritrócitos, hemoglobina e hematócrito após o término da prova em todos os cavalos, e esse aumento se manteve até três horas após a prova nos cavalos de 120 e $80 \mathrm{~km}$. Houve aumento do número de plaquetas em todos os cavalos, que permaneceu elevado até três horas após a prova e normalizou-se a partir do terceiro dia. Houve efeito $(\mathrm{P}<0,05)$ da prova sobre as variáveis eritrócitos, hemoglobina e hematócrito, do número de plaquetas, aumento na contagem de neutrófilos e diminuição da contagem de linfócitos após o término da prova e três horas depois, retornando ao normal a partir do terceiro dia. Um dado inédito deste estudo foi o aumento de eosinófilos sanguíneos após três dias da competição, que se manteve até o sétimo dia. Esse fato pode sinalizar a participação dessas células no processo de reparação. A avaliação dos componentes sanguíneos se mostrou uma importante ferramenta para a avaliação da recuperação dos animais. Como só foram avaliados cavalos que completaram a prova, as alterações encontradas após dias do esforço sinalizam a relevância de se estudarem melhor as etapas e os componentes da recuperação metabólica e muscular, a fim de não se cometerem excessos, retornando esses animais no tempo correto.

Palavras-chave: cavalos de enduro, hemograma, pós-prova, recuperação

\begin{abstract}
The aim of this study was to analyze the hematological changes after endurance races from different distances up to 14 days, a period of metabolic and musculoskeletal recovery that occured in trained horses that finished the competitions in a good health. A total of 32 Arabian horses were evaluated, with $400( \pm 32 \mathrm{~kg})$ trained for endurance races, who competed in four events in free speed categories $80 \mathrm{~km}(\mathrm{n}$ $=13), 120 \mathrm{~km}(n=14)$ and $160 \mathrm{~km}(n=5)$. Blood samples were collected before the start $(M 0)$, immediately after leaving the last veterinary inspection (M1) and three hours later (M2). Already at their farms, samples were taken at three (M3), seven (M4) and 14 (M5) days after the race. There was an increase of values of erythrocytes, hemoglobin and packed cell volume after the end of the race in all horses and this increase was maintained until three hours after the race in the of 120 and $80 \mathrm{~km}$ horses. There was an increase in the number of platelets in all horses, which remained elevated up to 3 hours after the test and normalized on the third day. All horses underwent increase in neutrophil counts and decreased lymphocyte count immediately after the race and three hours later, returning to normal after the third day. There was also pronounced increase in the number of eosinophils in all horses on the third
\end{abstract}

Recebido em 19 de setembro de 2016

Aceito em 3 de dezembro de 2016

*E-mail: refarinelli@yahoo.com.br 
day, which lasted until the seventh after the race. An unheard of data of this study was an increase in blood eosinophils after three days of competition that remained until the seventh day. This may signal the participation of these cells in the repair process. The evaluation of blood components proved to be an important tool for the assessment of recovery of the animals. As only horses that finished the race were evaluated, the changes found within days of effort, indicate the importance of further studies to investigate the steps and components of the metabolic and muscular recovery, in order not to commit excesses, returning the animals to activity at the right time.

Keywords: endurance horse, hemogram, post race, recovery

\section{INTRODUÇÃO}

O enduro equestre é um esporte amador praticado em 61 países, contando com cerca de 40 mil competidores, segundo dados fornecidos pela Confederação Brasileira de Hipismo (CBH). As provas de velocidade livre são divididas em três categorias, 80,120 e $160 \mathrm{~km}$, adultos, que correm com $75 \mathrm{~kg}$ de peso mínimo e young riders, cavaleiros de até 21 anos que correm sem peso.

Esse esporte, apesar de ainda amador, está se profissionalizando, e as pessoas envolvidas têm procurado mais por informações. Com a entrada dos povos árabes no esporte, criou-se um mercado de cavalos enduristas, e as médias de velocidade crescem a cada dia. Existe uma crescente preocupação com o bem-estar desses cavalos, com métodos de treinamento e suplementação, mas pouco se sabe sobre o que realmente acontece durante e após as provas. A literatura sobre o assunto é escassa.

Muito se estuda sobre exercício de resistência em cavalos, mas a maioria desses trabalhos tem enfoque em desidratação, metabolismo energético e estresse oxidativo (Robert et al., 2002; McKeever, 2004; Schott et al., 2006; Barnes et al., 2010). Poucos estudos são realizados a campo, onde há influência direta do cavaleiro, do terreno e do clima. Também muito pouco se sabe sobre a recuperação no período pós-prova, que, em humanos, é bem estudada, e sabe-se que é crucial para a recuperação muscular, a regeneração de glicogênio e é um período de imunossupressão, quando alguns atletas apresentam infecções até 14 dias depois de provas de resistência extenuantes (Gleeson, 2007; Lippi et al., 2010).

O hemograma é uma importante ferramenta para o auxílio no diagnóstico de enfermidades na clínica médica equina, além de também ser útil para avaliar aspectos da capacidade atlética do animal. O conhecimento dos constituintes sanguíneos representa a base para a avaliação das alterações patológicas, facilitando o diagnóstico de diferentes tipos de problemas nos equinos.

O objetivo deste trabalho foi estudar as alterações hematológicas após provas de enduro de velocidade livre de diferentes distâncias (80, 120 e $160 \mathrm{~km}$ ) até 14 dias após a prova, período de recuperação metabólica e musculoesquelética, as quais ocorrem em cavalos treinados e que concluíram as competições com aprovação na última inspeção veterinária.

\section{MATERIAL E MÉTODOS}

Este estudo foi aprovado pela Comissão de Ética de Uso de Animais (Ceua) da Faculdade de Medicina Veterinária e Zootecnia da Universidade de São Paulo, com o número de protocolo 2606/2012.

Foram utilizados 32 cavalos da raça Árabe, com $400( \pm 32 \mathrm{~kg})$, treinados para provas de enduro, pertencentes a proprietários enduristas, alojados em centros de treinamentos e haras particulares nas cidades de Bragança Paulista, Itatiba, Salesópolis, Jundiaí e Sorocaba, no estado de São Paulo. Esses animais foram avaliados em quatro provas chanceladas pela FEI (Fédération Equestre Internationale) e pela $\mathrm{CBH}$ (Confederação Brasileira de Hipismo). Cavalos eliminados tanto por problemas metabólicos quanto por claudicação não foram considerados. Somente os que receberam aprovação na inspeção veterinária final foram incluídos neste estudo, em que 13 cavalos competiram na categoria velocidade livre de $80 \mathrm{~km}, 14$ na de $120 \mathrm{~km}$ e cinco na de $160 \mathrm{~km}$.

Os tempos de colheita foram no dia da competição, antes da largada (M0), imediatamente após a saída da última inspeção 
veterinária (M1) e três horas após o término da prova (M2). Após o regresso dos animais às propriedades de origem, amostras de sangue foram coletadas nos dias três (M3), sete (M4) e 14 (M5) após a prova.

Amostras de sangue foram coletadas por venopunção jugular em tubos de coleta a vácuo com EDTA para a realização do hemograma. As amostras foram refrigeradas e encaminhadas ao laboratório da Faculdade de Medicina Veterinária e Zootecnia da Universidade de São Paulo. A análise do hemograma foi realizada no contador automático BC 2800Vet (Mindray, EUA).

As lâminas para diferencial dos leucócitos foram confeccionadas na hora da coleta de sangue, no próprio local, para que não houvesse alteração celular, coradas pelo método de May-GriinwaldGiemsa modificado e lidas no laboratório.

Os resultados foram submetidos ao teste de normalidade Kolmogorov-Smirnov e, posteriormente, à análise de variância por ANOVA e ao teste de comparação de Tukey, com 5\% de significância, e, para tal, foi utilizado o programa estatístico Minitab $17^{\circledR}$.

\section{RESULTADOS E DISCUSSÃO}

A média de velocidade, nas quatro provas em que os cavalos competiram, foi $17.56( \pm 1.3)$ para a categoria de $160 \mathrm{~km}, 18.42( \pm 1.4)$ para a de $120 \mathrm{~km}$ e $20.64( \pm 1.6)$ para a de $80 \mathrm{~km}$, havendo diferença entre as categorias $(\mathrm{P}=0,001)$.

Houve efeito da prova $(\mathrm{P}<0,05)$ sobre as variáveis eritrócitos, hemoglobina, hematócrito e do número de plaquetas após o término da competição em todos os cavalos, e esse aumento se manteve até três horas após a prova nos cavalos de 120 e $80 \mathrm{~km}$, conforme se observa na Tab. 1.

Os valores de eritrócitos, hemoglobina e hematócrito aumentaram após o término da prova em todos os cavalos, e esse aumento se manteve até três horas após a prova nos cavalos de 120 e $80 \mathrm{~km}$. Caso se considere que a velocidade com que esses cavalos competiram foi mais alta que a dos cavalos de $160 \mathrm{~km}$, podese sugerir que a necessidade de maior oxigenação da musculatura nos cavalos de enduro tenha mais relação com a intensidade do que com a duração do esforço, o que corrobora os achados de Rose et al. (1983), que concluíram que a contração esplênica é esforço-dependente.

Orozco et al. (2006) estudaram o efeito do exercício sobre variáveis hematológicas de equinos antes e após participação em prova de enduro de $40 \mathrm{~km}$ e encontraram aumento somente do hematócrito; as outras variáveis permaneceram estáveis. Já Teixeira Neto (2002) observou elevação do hematócrito, da concentração de hemoglobina e do número total de hemáceas ao final do percurso de $60 \mathrm{~km}$. Em outras modalidades equestres, Rubio et al. (1996) avaliaram a influência dos exercícios de trote e galope sobre o eritrograma de garanhões Lusitanos e observaram que o aumento do número de eritrócitos, hemoglobina e hematócrito após o exercício de galope foi maior que do exercício de trote e que os parâmetros normalizaram mais rápido nos exercícios de trote. Krumrych (2006) estudou a variação hematológica de cavalos de salto após exercício e também observou aumento dos valores de eritrócitos e hemoglobina após o exercício, com normalização 30 minutos depois. Kowal et al. (2006) avaliaram cavalos Puro Sangue Inglês de corrida submetidos a teste de esforço em esteira ergométrica e observaram que os valores referentes aos parâmetros hematológicos (contagem total de hemáceas, hematócrito e hemoglobina) aumentaram conforme a intensidade do exercício, embora 10 minutos depois do término do teste tenha havido reduções nos valores desses parâmetros. Zobba et al. (2011) estudaram cavalos de polo após um jogo internacional e encontraram as mesmas respostas descritas: aumento dos valores de eritrócitos, hemoglobina e hematócrito. 
Tabela 1. Média e desvio-padrão de variáveis hematológicas nos diferentes momentos de coleta de cavalos de enduro que competiram em 160, 120 e $80 \mathrm{~km}$

\begin{tabular}{|c|c|c|c|c|c|c|c|c|c|}
\hline & $\mathrm{N}$ & $\begin{array}{l}\text { Eritrócitos } \\
\left(10^{6} / \mathrm{mm}^{3}\right)\end{array}$ & $\begin{array}{c}\text { Hemoglobina } \\
(\mathrm{g} / \mathrm{dL})\end{array}$ & $\begin{array}{c}\text { Hematócrito } \\
(\%)\end{array}$ & $\begin{array}{l}\text { Plaquetas } \\
\left(10^{3} / \mathrm{mm}^{3}\right)\end{array}$ & $\begin{array}{l}\text { VCM } \\
\text { (fL) }\end{array}$ & $\begin{array}{c}\mathrm{HCM} \\
(\mathrm{pg})\end{array}$ & $\begin{array}{l}\text { CHCM } \\
(\mathrm{g} / \mathrm{dL})\end{array}$ & $\begin{array}{c}\text { RDW } \\
(\%)\end{array}$ \\
\hline $\begin{array}{l}160 \\
\mathrm{~km}\end{array}$ & 5 & & & & & & & & \\
\hline M0 & & $\begin{array}{r}7,17^{\mathrm{B}} \\
(1,03)\end{array}$ & $\begin{array}{l}13,52^{\mathrm{B}} \\
(2,08)\end{array}$ & $\begin{array}{l}37,30^{\mathrm{B}} \\
(3,00)\end{array}$ & $\begin{array}{c}171,80^{\mathrm{B}} \\
(66,7)\end{array}$ & $\begin{array}{l}52,16 \\
(2,89)\end{array}$ & $\begin{array}{l}18,80 \\
(0,78)\end{array}$ & $\begin{array}{l}36,14 \\
(1,34)\end{array}$ & $\begin{array}{l}17,74 \\
(0,85)\end{array}$ \\
\hline M1 & & $\begin{array}{l}8,02^{\mathrm{A}} \\
(1,01)\end{array}$ & $\begin{array}{l}15,14^{\mathrm{A}} \\
(1,54)\end{array}$ & $\begin{array}{l}41,56^{\mathrm{A}} \\
(3,70)\end{array}$ & $\begin{array}{c}257,40^{\mathrm{A}} \\
(110,3)\end{array}$ & $\begin{array}{l}52,10 \\
(3,33)\end{array}$ & $\begin{array}{l}18,88 \\
(0,78)\end{array}$ & $\begin{array}{l}36,34 \\
(1,14)\end{array}$ & $\begin{array}{l}17,56 \\
(0,85)\end{array}$ \\
\hline M2 & & $\begin{array}{l}7,53^{\mathrm{B}} \\
(0,72)\end{array}$ & $\begin{array}{c}14,08^{\mathrm{A}, \mathrm{B}} \\
(1,22)\end{array}$ & $\begin{array}{c}38,48^{\mathrm{B}} \\
(2,92)\end{array}$ & $\begin{array}{c}215,60^{\mathrm{A}} \\
(81,7)\end{array}$ & $\begin{array}{l}51,30 \\
(3,07)\end{array}$ & $\begin{array}{l}18,68 \\
(0,66)\end{array}$ & $\begin{array}{l}40,54 \\
(3,82)\end{array}$ & $\begin{array}{l}17,68 \\
(0,86)\end{array}$ \\
\hline M3 & & $\begin{array}{l}8,33^{\mathrm{A}} \\
(1,49)\end{array}$ & $\begin{array}{l}16,12^{\mathrm{C}} \\
(2,55)\end{array}$ & $\begin{array}{c}39,26^{A, B} \\
(4,13)\end{array}$ & $\begin{array}{c}164,80^{\mathrm{B}} \\
(75,2)\end{array}$ & $\begin{array}{l}51,30 \\
(3,07)\end{array}$ & $\begin{array}{l}19,14 \\
(1,22)\end{array}$ & $\begin{array}{l}37,40 \\
(1,99)\end{array}$ & $\begin{array}{l}16,64 \\
(1,35)\end{array}$ \\
\hline M4 & & $\begin{array}{c}7,60^{\mathrm{B}} \\
(1,80)\end{array}$ & $\begin{array}{l}13,84^{\mathrm{B}} \\
(2,17)\end{array}$ & $\begin{array}{c}36,88^{\mathrm{B}} \\
(4,23)\end{array}$ & $\begin{array}{c}206,80^{\mathrm{A}} \\
(128,7)\end{array}$ & $\begin{array}{l}52,04 \\
(1,98)\end{array}$ & $\begin{array}{l}18,44 \\
(1,09)\end{array}$ & $\begin{array}{l}35,54 \\
(1,87)\end{array}$ & $\begin{array}{l}16,12 \\
(1,22)\end{array}$ \\
\hline M5 & & $\begin{array}{l}7,57^{\mathrm{B}} \\
(1,74)\end{array}$ & $\begin{array}{l}14,60^{\mathrm{A}} \\
(2,90)\end{array}$ & $\begin{array}{c}37,52^{\mathrm{B}} \\
(2,61)\end{array}$ & $\begin{array}{c}135,40^{\mathrm{C}} \\
(24,1)\end{array}$ & $\begin{array}{l}51,98 \\
(2,29) \\
\end{array}$ & $\begin{array}{l}19,26 \\
(1,26)\end{array}$ & $\begin{array}{l}37,12 \\
(2,95)\end{array}$ & $\begin{array}{l}16,12 \\
(1,12)\end{array}$ \\
\hline $\begin{array}{l}120 \\
\mathrm{~km}\end{array}$ & 14 & & & & & & & & \\
\hline M0 & & $\begin{array}{c}7,70^{B, C} \\
(0,90)\end{array}$ & $\begin{array}{c}14,32^{\mathrm{B}, \mathrm{C}} \\
(1,63)\end{array}$ & $\begin{array}{c}39,50^{\mathrm{B}, \mathrm{C}} \\
(2,85)\end{array}$ & $\begin{array}{c}181,30^{\mathrm{A}, \mathrm{B}} \\
(63,5)\end{array}$ & $\begin{array}{l}51,57 \\
(2,52)\end{array}$ & $\begin{array}{l}18,57 \\
(0,85)\end{array}$ & $\begin{array}{l}36,18 \\
(1,56)\end{array}$ & $\begin{array}{l}17,20 \\
(1,05)\end{array}$ \\
\hline M1 & & $\begin{array}{l}8,84^{\mathrm{A}} \\
(0,96)\end{array}$ & $\begin{array}{l}16,53^{\mathrm{A}} \\
(1,55)\end{array}$ & $\begin{array}{l}45,73^{\mathrm{A}} \\
(2,25)\end{array}$ & $\begin{array}{c}227,70^{\mathrm{A}} \\
(90,1)\end{array}$ & $\begin{array}{l}51,92 \\
(2,24)\end{array}$ & $\begin{array}{l}18,70 \\
(0,92)\end{array}$ & $\begin{array}{l}36,12 \\
(1,17)\end{array}$ & $\begin{array}{l}17,07 \\
(0,70)\end{array}$ \\
\hline M2 & & $\begin{array}{c}8,33^{\mathrm{A}, \mathrm{B}} \\
(1,00)\end{array}$ & $\begin{array}{c}15,37^{\mathrm{A}, \mathrm{B}} \\
(1,73)\end{array}$ & $\begin{array}{c}42,39^{\mathrm{A}, \mathrm{B}} \\
(2,96)\end{array}$ & $\begin{array}{c}223,90^{\mathrm{A}} \\
(65,3)\end{array}$ & $\begin{array}{l}51,15 \\
(2,50)\end{array}$ & $\begin{array}{l}18,44 \\
(1,03)\end{array}$ & $\begin{array}{l}36,15 \\
(1,32)\end{array}$ & $\begin{array}{l}17,33 \\
(0,93)\end{array}$ \\
\hline M3 & & $\begin{array}{l}7,25^{\mathrm{C}} \\
(0,84)\end{array}$ & $\begin{array}{c}13,58^{\mathrm{B}, \mathrm{C}, \mathrm{D}} \\
(1,85)\end{array}$ & $\begin{array}{c}37,18^{\mathrm{C}, \mathrm{D}} \\
(2,48)\end{array}$ & $\begin{array}{l}145,50^{\mathrm{B}} \\
(34,04)\end{array}$ & $\begin{array}{l}51,33 \\
(2,03)\end{array}$ & $\begin{array}{l}18,68 \\
(1,19)\end{array}$ & $\begin{array}{l}36,15 \\
(1,65)\end{array}$ & $\begin{array}{l}16,06 \\
(1,19)\end{array}$ \\
\hline M4 & & $\begin{array}{l}6,77^{\mathrm{C}} \\
(0,78)\end{array}$ & $\begin{array}{l}12,32^{\mathrm{D}} \\
(1,27)\end{array}$ & $\begin{array}{c}34,44^{\mathrm{C}, \mathrm{D}} \\
(2,31)\end{array}$ & $\begin{array}{c}168,70^{\mathrm{A}, \mathrm{B}} \\
(39,00)\end{array}$ & $\begin{array}{l}51,08 \\
(2,91)\end{array}$ & $\begin{array}{l}18,21 \\
(1,06)\end{array}$ & $\begin{array}{l}36,50 \\
(1,61)\end{array}$ & $\begin{array}{l}16,31 \\
(1,39)\end{array}$ \\
\hline M5 & & $\begin{array}{l}7,10^{\mathrm{C}} \\
(0,66)\end{array}$ & $\begin{array}{c}13,43^{\mathrm{C}, \mathrm{D}} \\
(1,84)\end{array}$ & $\begin{array}{l}36,20^{D} \\
(2,36)\end{array}$ & $\begin{array}{l}154,93^{\mathrm{B}} \\
(31,64)\end{array}$ & $\begin{array}{l}51,97 \\
(2,52)\end{array}$ & $\begin{array}{l}19,01 \\
(2,16)\end{array}$ & $\begin{array}{l}36,65 \\
(1,67)\end{array}$ & $\begin{array}{l}16,03 \\
(1,16)\end{array}$ \\
\hline $\begin{array}{r}80 \\
\mathrm{~km} \\
\end{array}$ & 13 & & & & & & & & \\
\hline M0 & & $\begin{array}{l}7,97^{\mathrm{B}, \mathrm{C}} \\
(1,19)\end{array}$ & $\begin{array}{l}13,36 \\
(1,28)\end{array}$ & $\begin{array}{c}39,16^{\mathrm{B}, \mathrm{C}} \\
(2,47)\end{array}$ & $\begin{array}{c}347,40^{\mathrm{A}} \\
(159,4)\end{array}$ & $\begin{array}{l}49,45 \\
(2,23)\end{array}$ & $\begin{array}{l}16,98 \\
(2,35)\end{array}$ & $\begin{array}{l}34,30 \\
(2,17)\end{array}$ & $\begin{array}{l}17,52 \\
(0,93)\end{array}$ \\
\hline M1 & & $\begin{array}{l}9,22^{\AA} \\
(0,82)\end{array}$ & $\begin{array}{l}15,72 \\
(1,63)\end{array}$ & $\begin{array}{r}45,76^{\mathrm{A}} \\
(1,69)\end{array}$ & $\begin{array}{c}404,90^{\mathrm{B}} \\
(151,3)\end{array}$ & $\begin{array}{l}49,76 \\
(2,14)\end{array}$ & $\begin{array}{l}17,08 \\
(2,34)\end{array}$ & $\begin{array}{l}34,34 \\
(2,12)\end{array}$ & $\begin{array}{l}17,62 \\
(0,83)\end{array}$ \\
\hline M2 & & $\begin{array}{c}8,56^{\mathrm{A}, \mathrm{B}} \\
(1,09)\end{array}$ & $\begin{array}{l}14,56 \\
(1,43)\end{array}$ & $\begin{array}{c}42,12^{\mathrm{A}, \mathrm{B}} \\
(2,25)\end{array}$ & $\begin{array}{l}407,20^{\mathrm{B}} \\
(160,5)\end{array}$ & $\begin{array}{l}49,25 \\
(2,06)\end{array}$ & $\begin{array}{l}17,10 \\
(2,13)\end{array}$ & $\begin{array}{l}34,70 \\
(2,97)\end{array}$ & $\begin{array}{l}17,60 \\
(0,83)\end{array}$ \\
\hline M3 & & $\begin{array}{l}7,27^{\mathrm{B}, \mathrm{C}} \\
(1,05)\end{array}$ & $\begin{array}{l}13,60 \\
(2,69)\end{array}$ & $\begin{array}{c}38,00^{\mathrm{B}, \mathrm{C}} \\
(2,98)\end{array}$ & $\begin{array}{c}211,80^{\mathrm{C}} \\
(118,4)\end{array}$ & $\begin{array}{l}48,15 \\
(2,74)\end{array}$ & $\begin{array}{l}17,20 \\
(2,81)\end{array}$ & $\begin{array}{l}35,68 \\
(2,34)\end{array}$ & $\begin{array}{l}16,83 \\
(1,26)\end{array}$ \\
\hline M4 & & $\begin{array}{c}7,37^{\mathrm{C}} \\
(0,87)\end{array}$ & $\begin{array}{l}12,09 \\
(1,50)\end{array}$ & $\begin{array}{l}35,33^{\mathrm{C}} \\
(3,05)\end{array}$ & $\begin{array}{l}208,5^{\mathrm{C}} \\
(111,1)\end{array}$ & $\begin{array}{l}48,87 \\
(2,39)\end{array}$ & $\begin{array}{l}16,78 \\
(2,24)\end{array}$ & $\begin{array}{l}34,24 \\
(2,43)\end{array}$ & $\begin{array}{l}16,73 \\
(0,97)\end{array}$ \\
\hline M5 & & $\begin{array}{c}7,48^{\mathrm{B}, \mathrm{C}} \\
(0,82)\end{array}$ & $\begin{array}{l}12,80 \\
1,79)\end{array}$ & $\begin{array}{l}35,80^{\mathrm{C}} \\
(2,62) \\
\end{array}$ & $\begin{array}{l}208,4^{\mathrm{C}} \\
(114,6)\end{array}$ & $\begin{array}{l}49,36 \\
(2,03) \\
\end{array}$ & $\begin{array}{l}17,78 \\
(2,64) \\
\end{array}$ & $\begin{array}{l}35,82 \\
(2,58) \\
\end{array}$ & $\begin{array}{l}16,70 \\
(1,03) \\
\end{array}$ \\
\hline
\end{tabular}

Letras diferentes na mesma coluna indicam diferença da variável entre os diferentes momentos.

Houve também aumento do número de plaquetas em todos os cavalos, que permaneceu elevado até três horas após a prova e normalizou-se a partir do terceiro dia. Alguns estudos em humanos demonstraram aumento de 18 a $80 \%$ do número de plaquetas após sessões de exercício na esteira e em bicicleta, de acordo com o esforço (Warlow, Ogston, 1974; Metha, Metha, 1982; Davis et al., 1990; Wang et al., 1994). O exercício físico exerce efeito sobre vários fatores 
da coagulação e fibrinolíticos, bem como sobre a função das plaquetas no sangue. Curiosamente, as sessões agudas de exercício produzem diferentes efeitos na homeostasia em comparação com os efeitos produzidos pelo treinamento físico (exercício crônico). A resposta fisiológica imediata a uma sessão aguda de exercício é caracterizada por um estado de hipercoagulabilidade transitória, com encurtamento do tempo de ativação da tromboplastina e aumento da geração de trombina, elevação nos níveis plasmáticos do fator de coagulação VIII e fator de Von Willebrand, assim como aumento na contagem de plaquetas e reatividade plaquetária (Sossdorf et al., 2011).

Quanto às células brancas, todos os cavalos sofreram aumento na contagem de neutrófilos e diminuição na contagem de linfócitos imediatamente após a prova e três horas depois, retornando ao normal a partir do terceiro dia, conforme ilustra a Tab. 2.

O esforço físico prolongado e de alta intensidade causa alteração bifásica na contagem de células brancas na circulação, por modificações hormonais, concentração de citocinas, temperatura corporal, aumento do fluxo sanguíneo, vasoconstrição e desidratação. A adrenalina aumentada durante o exercício recruta linfócitos, principalmente do baço, onde existe o maior estoque dessas células para o sangue, porém sua ação é atenuada pelo aumento do cortisol no fim do exercício (Nielman, 1997). O cortisol aumenta a liberação de neutrófilos da medula óssea na circulação, e o aumento do débito cardíaco provoca turbilhonamento do sangue, aumentando a mobilização de leucócitos de compartimentos marginais como parede das veias (Robson et al., 2003, Risøy et al., 2003). No presente estudo, houve neutrofilia no pósprova e linfopenia, que persistiu até o $14^{\circ}$ dia nos cavalos de $160 \mathrm{~km}$.

Outros autores (Robson et al., 2003; Cywińska et al., 2010; Cywińska et al., 2012) estudaram cavalos de enduro de regularidade, velocidade livre curta e longa distância e todos encontraram a mesma alteração. Robson et al. (2003) encontraram neutrofilia e linfopenia evidentes uma hora e um dia após enduro de $80 \mathrm{~km}$, retornando à normalidade somente três dias após, como ocorreu no presente estudo. Alguns autores (Kurcz et al., 1998; Lunn et al., 2001), que trabalharam com cavalos em esteira rolante de alta velocidade, encontraram supressão da resposta proliferativa de linfócitos, porém Cywińska et al. (2012) encontraram diminuição dessa resposta somente em cavalos de longa distância $(160 \mathrm{~km})$ e não nos cavalos de regularidade.

Houve pronunciado aumento do número de eosinófilos em todos os cavalos deste estudo no terceiro dia, que durou até o sétimo após a prova. Eosinófilos são granulócitos produzidos na medula óssea e acredita-se que para cada célula presente na circulação haja de 100-300 células nos tecidos. Produzem diferentes tipos de proteínas em seus grânulos responsáveis pela sua ação nos patógenos e produzem também algumas citocinas, como IL-2, IL-6, IL-8, TGF $\alpha$ e TGF $\beta$, que agem como fatores de crescimento para outros eosinófilos e ativação de células maduras, o que potencialmente interfere nos processos inflamatórios (Venge, 1998).

Tanto o exercício de resistência quanto o de força em humanos podem ativar eosinófilos por via não alérgica, pois essas células também participam do processo de regeneração da musculatura lesionada por meio da modificação da produção de glicosaminoglicanos pelos fibroblastos. Outra possível explicação é que a resposta de fase aguda na musculatura lesionada ativa o sistema complemento $(\mathrm{C} 3, \mathrm{C} 4 \mathrm{e}$ C5) e esses fragmentos podem ativar os eosinófilos (Dufaux et al., 1993). McKune et al. (2004) estudaram concentrações séricas de proteína eosinofílica catiônica (ECP) em ultramaratonistas humanos e perceberam que tanto a ECP quanto o número de eosinófilos permaneceram alterados após 72 horas da prova, exatamente como ocorreu no presente estudo, embora nos cavalos a eosinofilia tenha persistido até o sétimo dia, talvez por possuírem maior massa muscular que os humanos. Não há relatos em equinos de eosinofilia pós-exercício nem que dure tanto tempo. 
Tabela 2. Média e desvio-padrão de variáveis do leucograma nos diferentes momentos de coleta de cavalos de enduro que competiram em 160,120 e $80 \mathrm{~km}$

\begin{tabular}{|c|c|c|c|c|c|c|c|}
\hline & $\mathrm{N}$ & $\begin{array}{l}\text { Leucócitos } \\
\text { Totais } \\
\left(10^{3} / \mathrm{mm}^{3}\right) \\
\end{array}$ & $\begin{array}{c}\text { Neutrófilos } \\
\left(10^{3} / \mathrm{mm}^{3}\right)\end{array}$ & $\begin{array}{l}\text { Linfócitos } \\
\left(10^{3} / \mathrm{mm}^{3}\right)\end{array}$ & $\begin{array}{l}\text { Eosinófilos } \\
\left(10^{3} / \mathrm{mm}^{3}\right)\end{array}$ & $\begin{array}{l}\text { Monócitos } \\
\left(10^{3} / \mathrm{mm}^{3}\right)\end{array}$ & $\begin{array}{l}\text { Basófilos } \\
\left(10^{3} / \mathrm{mm}^{3}\right)\end{array}$ \\
\hline $\begin{array}{l}160 \\
\mathrm{~km}\end{array}$ & 5 & & & & & & \\
\hline M0 & & $\begin{array}{c}11,08^{\mathrm{A}, \mathrm{B}, \mathrm{C}} \\
(3,74)\end{array}$ & $\begin{array}{l}5,74^{\mathrm{B}} \\
(2,90)\end{array}$ & $\begin{array}{l}4,82^{\mathrm{B}} \\
(2,49)\end{array}$ & $\begin{array}{l}0,16^{\mathrm{B}} \\
(0,19)\end{array}$ & $\begin{array}{c}0,08 \\
(0,11)\end{array}$ & $\begin{array}{c}0,16 \\
(0,25)\end{array}$ \\
\hline M1 & & $\begin{array}{l}14,60^{\mathrm{A}} \\
(3,21)\end{array}$ & $\begin{array}{l}12,47^{\mathrm{A}} \\
(2,65)\end{array}$ & $\begin{array}{l}1,90^{\mathrm{A}} \\
(0,80)\end{array}$ & $\begin{array}{l}0,04^{\mathrm{A}} \\
(0,10)\end{array}$ & $\begin{array}{c}0,02 \\
(0,06)\end{array}$ & $\begin{array}{c}0,16 \\
(0,25)\end{array}$ \\
\hline M2 & & $\begin{array}{c}13,88^{A, B} \\
(3,31)\end{array}$ & $\begin{array}{l}11,78^{\mathrm{A}} \\
(2,48)\end{array}$ & $\begin{array}{l}2,10^{\mathrm{A}} \\
(0,42)\end{array}$ & $\begin{array}{l}0,07^{\mathrm{A}} \\
(0,12)\end{array}$ & $\begin{array}{c}0,00 \\
(0,00)\end{array}$ & $\begin{array}{c}0,12 \\
(0,10)\end{array}$ \\
\hline M3 & & $\begin{array}{l}6,58^{\mathrm{C}} \\
(2,19)\end{array}$ & $\begin{array}{l}3,67^{\mathrm{B}} \\
(1,62)\end{array}$ & $\begin{array}{l}2,71^{\mathrm{C}} \\
(1,17)\end{array}$ & $\begin{array}{l}0,25^{\mathrm{C}} \\
(0,17)\end{array}$ & $\begin{array}{c}0,02 \\
(0,04)\end{array}$ & $\begin{array}{c}0,10 \\
(0,11)\end{array}$ \\
\hline M4 & & $\begin{array}{c}7,56^{\mathrm{B}, \mathrm{C}} \\
(1,50)\end{array}$ & $\begin{array}{l}4,61^{\mathrm{B}} \\
(0,70)\end{array}$ & $\begin{array}{l}2,74^{\mathrm{C}} \\
(1,03)\end{array}$ & $\begin{array}{l}0,23^{\mathrm{C}} \\
(0,11)\end{array}$ & $\begin{array}{c}0,00 \\
(0,00)\end{array}$ & $\begin{array}{c}0,06 \\
(0,07)\end{array}$ \\
\hline M5 & & $\begin{array}{c}7,44^{\mathrm{B}, \mathrm{C}} \\
(0,81)\end{array}$ & $\begin{array}{l}4,30^{\mathrm{B}} \\
(0,60)\end{array}$ & $\begin{array}{l}3,03^{\mathrm{C}} \\
(0,84)\end{array}$ & $\begin{array}{l}0,08^{\mathrm{A}} \\
(0,05)\end{array}$ & $\begin{array}{c}0,03 \\
(0,07) \\
\end{array}$ & $\begin{array}{c}0,10 \\
(0,03)\end{array}$ \\
\hline $\begin{array}{l}120 \\
\mathrm{~km}\end{array}$ & 14 & & & & & & \\
\hline M0 & & $\begin{array}{l}9,26^{\mathrm{B}} \\
(2,88)\end{array}$ & $\begin{array}{l}5,23^{\mathrm{B}} \\
(1,73)\end{array}$ & $\begin{array}{l}3,77^{\mathrm{B}} \\
(1,68)\end{array}$ & $\begin{array}{l}0,18^{\mathrm{B}} \\
(0,14)\end{array}$ & $\begin{array}{c}0,05 \\
(0,06)\end{array}$ & $\begin{array}{c}0,10 \\
(0,20)\end{array}$ \\
\hline M1 & & $\begin{array}{l}13,86^{\mathrm{A}} \\
(2,57)\end{array}$ & $\begin{array}{l}10,35^{\mathrm{A}} \\
(2,60)\end{array}$ & $\begin{array}{l}1,35^{\mathrm{A}} \\
(1,37)\end{array}$ & $\begin{array}{l}0,05^{\mathrm{B}} \\
(0,11)\end{array}$ & $\begin{array}{c}0,00 \\
(0,00)\end{array}$ & $\begin{array}{c}0,10 \\
(0,11)\end{array}$ \\
\hline M2 & & $\begin{array}{l}14,89^{\mathrm{A}} \\
(2,97)\end{array}$ & $\begin{array}{l}11,73^{\mathrm{A}} \\
(2,83)\end{array}$ & $\begin{array}{l}1,02^{\mathrm{A}} \\
(1,53)\end{array}$ & $\begin{array}{l}0,08^{\mathrm{B}} \\
(0,17)\end{array}$ & $\begin{array}{c}0,01 \\
(0,04)\end{array}$ & $\begin{array}{c}0,16 \\
(0,15)\end{array}$ \\
\hline M3 & & $\begin{array}{l}8,62^{\mathrm{B}} \\
(3,30)\end{array}$ & $\begin{array}{l}4,19^{\mathrm{B}} \\
(2,16)\end{array}$ & $\begin{array}{l}3,81^{\mathrm{B}} \\
(1,75)\end{array}$ & $\begin{array}{l}0,60^{\mathrm{A}} \\
(0,35)\end{array}$ & $\begin{array}{c}0,04 \\
(0,01)\end{array}$ & $\begin{array}{c}0,13 \\
(0,15)\end{array}$ \\
\hline M4 & & $\begin{array}{l}9,93^{\mathrm{B}} \\
(3,06)\end{array}$ & $\begin{array}{l}5,49^{\mathrm{B}} \\
(2,84)\end{array}$ & $\begin{array}{l}3,93^{\mathrm{B}} \\
(1,35)\end{array}$ & $\begin{array}{l}0,59^{\mathrm{A}} \\
(0,40)\end{array}$ & $\begin{array}{c}0,04 \\
(0,01)\end{array}$ & $\begin{array}{c}0,16 \\
(0,07)\end{array}$ \\
\hline M5 & & $\begin{array}{l}7,77^{\mathrm{B}} \\
(1,43) \\
\end{array}$ & $\begin{array}{l}4,15^{\mathrm{B}} \\
(0,93)\end{array}$ & $\begin{array}{l}3,38^{\mathrm{B}} \\
(0,87)\end{array}$ & $\begin{array}{l}0,17^{\mathrm{B}} \\
(0,08)\end{array}$ & $\begin{array}{c}0,05 \\
(0,09)\end{array}$ & $\begin{array}{c}0,13 \\
(0,05)\end{array}$ \\
\hline $\begin{array}{l}80 \\
\mathrm{~km}\end{array}$ & 13 & & & & & & \\
\hline M0 & & $\begin{array}{l}7,81^{\mathrm{B}} \\
(1,29)\end{array}$ & $\begin{array}{l}4,30^{\mathrm{B}} \\
(1,05)\end{array}$ & $\begin{array}{l}3,43^{\mathrm{B}} \\
(0,78)\end{array}$ & $\begin{array}{l}0,07^{\mathrm{B}} \\
(0,07)\end{array}$ & $\begin{array}{c}0,02 \\
(0,04)\end{array}$ & $\begin{array}{c}0,12 \\
(0,08)\end{array}$ \\
\hline M1 & & $\begin{array}{l}12,85^{\mathrm{A}} \\
(2,02)\end{array}$ & $\begin{array}{l}10,41^{\mathrm{A}} \\
(3,19)\end{array}$ & $\begin{array}{l}1,42^{\mathrm{A}} \\
(0,66)\end{array}$ & $\begin{array}{l}0,04^{\mathrm{B}} \\
(0,09)\end{array}$ & $\begin{array}{c}0,08 \\
(0,03)\end{array}$ & $\begin{array}{l}0,15 \\
0,11)\end{array}$ \\
\hline M2 & & $\begin{array}{l}15,72^{\mathrm{A}} \\
(3,14)\end{array}$ & $\begin{array}{l}12,37^{\mathrm{A}} \\
(3,94)\end{array}$ & $\begin{array}{l}1,01^{A} \\
(1,32)\end{array}$ & $\begin{array}{l}0,16^{\mathrm{C}} \\
(0,51)\end{array}$ & $\begin{array}{c}0,02 \\
(0,06)\end{array}$ & $\begin{array}{c}0,13 \\
(0,22)\end{array}$ \\
\hline M3 & & $\begin{array}{l}7,05^{\mathrm{B}} \\
(2,08)\end{array}$ & $\begin{array}{c}3,79^{\mathrm{B}} \\
(1,78)\end{array}$ & $\begin{array}{l}2,46^{\mathrm{C}} \\
(1,24)\end{array}$ & $\begin{array}{l}0,57^{\mathrm{A}} \\
(0,24)\end{array}$ & $\begin{array}{c}0,01 \\
(0,02)\end{array}$ & $\begin{array}{c}0,17 \\
(0,11)\end{array}$ \\
\hline M4 & & $\begin{array}{l}7,30^{\mathrm{B}} \\
(1,38)\end{array}$ & $\begin{array}{l}4,26^{\mathrm{B}} \\
(1,14)\end{array}$ & $\begin{array}{l}2,70^{\mathrm{C}} \\
(0,63)\end{array}$ & $\begin{array}{l}0,31^{\mathrm{B}} \\
(0,13)\end{array}$ & $\begin{array}{c}0,02 \\
(0,02)\end{array}$ & $\begin{array}{c}0,13 \\
(0,05)\end{array}$ \\
\hline M5 & & $\begin{array}{l}7,22^{\mathrm{B}} \\
(1,33)\end{array}$ & $\begin{array}{c}4,10^{\mathrm{B}} \\
(1,17)\end{array}$ & $\begin{array}{l}2,85^{\mathrm{C}} \\
(0,59)\end{array}$ & $\begin{array}{c}0,18^{\mathrm{C}} \\
(0,11)\end{array}$ & $\begin{array}{c}0,04 \\
(0,07)\end{array}$ & $\begin{array}{c}0,14 \\
(0,07)\end{array}$ \\
\hline
\end{tabular}

Letras diferentes na mesma coluna indicam diferença da variável entre os diferentes momentos. 


\section{CONCLUSÕES}

A avaliação dos componentes sanguíneos se mostrou uma importante ferramenta para a avaliação da recuperação dos animais. Um achado relevante foi o pronunciado aumento do número de eosinófilos no sangue em todos os cavalos tardiamente. Como eosinófilos são células teciduais, tal aumento pode sinalizar que essas células possam servir de marcador de recuperação de esforço muscular. Como só foram avaliados cavalos que completaram a prova, as alterações encontradas após dias do esforço apontam a importância de se estudarem melhor as etapas e os componentes da recuperação metabólica e muscular, a fim de não se cometerem excessos, retornando esses animais no tempo correto.

\section{REFERÊNCIAS}

BARNES A.; KINGSTON J.; BEETSON S. et al. Endurance veterinarians detect physiologically compromised horses in a $160 \mathrm{~km}$ ride. Equine Vet. J, v. 42 (Suppl. 38), p.06-11, 2010.

CYWIŃSKA A.; SZARSKA E.; GÓRECKA R. et al. Lymphocyte proliferation activity after limited (Light class) and long (CEI) distance endurance rides in horses. Cent. Eur. J. Immunol., v.37, p.326-331, 2012.

CYWIŃSKA A.; WYSZYŃSKA Z; GÓRECKA R. et al. The effect of the $162 \mathrm{~km}$ endurance ride on equine peripheral blood neutrophil and lymphocyte functions. Pol. J. Vet. Sci., v.13, p.279-285, 2010.

DAVIS R.B.; BOYD D.G.; MCKINNEY M.E. et al. Effects of exercise and exercise conditioning on blood platelet function. Med. Sci. Sports Exerc., v.22, p.49-53, 1990.

DUFAUX B.; HEINE O.; PRINZ U. et al. Effect of short maximal physical exercise on the eosinophil cationic protein. Int. J. Sports Med., v.14, p.468-470, 1993.

GLEESON M. Immune function in sport and exercise. J. Appl. Physiol., v.103, n.2, p.693-699, 2007.
KOWAL R.J.; ALMOSNY N.R.P.; CASCARDO B. et al. Avaliação dos valores hematológicos em cavalos (Equus caballus) da raça Puro-Sangue-Inglês (PSI) submetidos a teste de esforço em esteira ergométrica. Rev. Bras. Ciênc. Vet., v.13, p.25-31, 2006.

KRUMRYCH W. Variability of clinical and hematological indices in the course of training exercise in jumping horses. Bull. Vet. Inst. Pulawy, v.50, p.391-396, 2006.

KURCZ E.V.; LAWRENCE L.M.; KELLEY K.W. et al. The effect of intense exercise on the cell-mediated immune response of horses. $J$. Equine Vet. Sci., v.8, p.237-239, 1998.

LIPPI G.; BANFI G.; MONTAGNANA M.; SALVAGNO L.G. et al. Acute variation of leukocytes counts following a half-marathon run. Int. J. Lab. Hematol., v.32, p.117-121, 2010.

LUNN D.P.; HUSSEY S.; SEBING R. et al. Safety, efficacy, and immunogenicity of a modified-live equine influenza virus vaccine in ponies after induction of exercise-induced immunosuppression. J. Am. Vet. Med. Assoc., v.15, p.900-906, 2001

McKEEVER K.H. Body fluids and electrolytes: Responses to exercise and training. In: (Eds.). HINCHCLIFF, K.W.; KANEPS, A.J.; GEOR, R.J. Equine sports medicine and surgery basic and clinical sciences of the equine athlete. Philadelphia: Saunders Elsevier, 2004. p.853871.

McKUNE A.J., SMITH L.L., SEMPLE S.J. et al. Non-allergic activation of eosinophils after strenuous endurance exercise. Sports Med., v.16, p.12-16, 2004.

MEHTA J., MEHTA P. Comparison of platelet function during exercise in normal subjects and coronary artery disease patients: potential role of platelet activation in myocardial ischemia. Am. Heart J., v.103, p.49-53, 1982.

NIEMAN, D.C. Risk of upper respiratory tract infection in athletes: an epidemiologic and immunologic perspective. J. Athletic Training, v.32, p.344-349, 1997. 
OROZOCO C.A.G.; MARTINS C.B.; D'ANGELIS F.H.F. et al. Efeito do exercício sobre variáveis hematológicas de equinos antes e após participação em prova de enduro de $40 \mathrm{~km}$. Ars Vet., v.22, p.179-183, 2006.

RISØY B.A.; RAASTAD T.; HALLÉN J. et al. Delayed leukocytosis after hard strength and endurance exercise: aspects of regulatory mechanisms. Biomed Cent. Physiol., v.3, p.1-12, 2003.

ROBERT, C.; BENAMOU-SMITH, A.; LECLERC, J.L. Use of the recovery check in long-distance endurance rides. Equine Vet. J. Suppl. v.34, p.106-111, 2002.

ROBSON P.J., ALSTON T.D., MYBURGH K. $H$. Prolonged suppression of the innate immune system in the horse following an $80 \mathrm{~km}$ endurance race. Equine Vet. J., v.35, p.133-137, 2003.

ROSE R.J.; ALLEN J.R, HODGSON D.R. et al. Responses to submaximal treadmill exercise and training in the horse: changes in haematology, arterial blood gas and acid base measurements, plasma biochemical values and heart rate. Vet. Rec., v.113, p.612-618, 1983.

ROSE R.J.; HODGSON D.R. Hematology and biochemistry. In: HODGSON, D.R.;

RUBIO M.D. et al. Influence of trotting and galloping exercises on erythrogram of andalusian horse stallions. J. Equine Vet. Sci., v.16, p.249251, 1996.
SCHOTT H.C.; MARLIN D.J.; GEOR, R.J. et al. Changes in selected physiological and laboratory measurements in elite horses competing in a $160 \mathrm{~km}$ endurance ride. Equine Vet. J. Suppl., v.36, p.37-42, 2006.

SOSSDORF M.; OTTO G.P.; CLAUS R.F. et al. Cell-derived microparticles promote coagulation after moderate exercise. Med. Sci. SportS Exerc., v.11, p.1169-1176, 2011.

TEIXEIRA NETO A.R. Efeitos da reposição eletrolítica sobre as variáveis fisiológicas de cavalos em provas de enduro de 30 e $60 \mathrm{~km}$ de distância. 2002. 92f. Dissertação (Mestrado em Clínica Médica Veterinária) - Universidade Estadual Paulista, Jaboticabal, SP.

VENGE, P. Eosinophisl. In: BARNES P.J.; RODGER I.W.; THOMSON N.C. Asthma: basic mechanisms and clinical management. 3ed. Oxford: Oxford University Press, 1998. p.142157.

WANG J.; JEN C.J.; KUNG H. et al. Different effects of strenuous exercise and moderate exercise on platelet function in men. Circulation, v.90, p.2877-2885, 1994.

WARLOW C.P.; OGSTON D. Effect of exercise on platelet count, adhesion, and aggregation. Acta Haematol., v.52, p.47-52, 1974.

ZOBBA, R. et al. Physical, hematological, and biochemical responses to acute intense exercise in polo horses. J. Equine Vet. Sci., v.31, p.542548, 2011. 